\title{
Molecular strategies for the electrophysiologist
}

\author{
Luca Boveri and Silvia G Priori
}

Since the release of results from the Cardiac Arrhythmia Suppression Trial (CAST), trust in pharmacological prevention of sudden cardiac death has progressively reduced. The search for approaches alternative to antiarrhythmic agents for the prevention of ventricular arrhythmias has led to the current situation in which catheter ablation and the implantation of defibrillators are the leading tools of the arrhythmia specialist.

Over the past decade, the rise of molecular biology has enlightened several areas of medicine, including cardiology, by providing a novel understanding of the physiology and substrates of different disease states. In this setting, it is hoped that the field of electrophysiology may be able to exploit such advancements for therapeutic purposes. Improved understanding of the biochemical mechanisms that cause cardiac electrical dysfunctions, together with advancements in the knowledge of the cellular and molecular basis of arrhythmias, has resulted in the identification of interesting molecules and metabolic pathways, which could be targeted or exploited for the modulation of rhythm disturbances. As of today, several groups have provided the "proof of concept', demonstrating that it is possible to use genetic manipulation to modify arrhythmogenic substrates in animal models as well as in vitro.

The application of gene therapy for the management of cardiac rhythm already seems promising in a few broad areas. One area is the restoration of specialized function in the heart by promoting overexpression of critical and tissue-specific ion channels. For example, major advancements in the development of a biological pacemaker have been made by forcing regional expression of $\mathrm{HCN} 4$ (hyperpolarization activated cyclic nucleotide-gated potassium channel 4), a gene that encodes the channel that conducts the pacemaker current $\left(I_{f}\right)$. The potential of this technique has been

\section{The application \\ of gene \\ therapy for \\ cardiac rhythm \\ management \\ already seems \\ promising...}

L Boveri is a Post

Doctoral Fellow at

the Department of

Molecular Cardiology,

IRCCS Fondazione

Salvatore Maugeri,

Pavia, Italy and

SG Priori is Director

of Molecular

Cardiology, University

of Pavia, and an

Advisory Board

Member of Nature

Clinical Practice

Cardiovascular

Medicine.

\section{Competing interests}

The authors declared no

competing interests.

www.nature.com/clinicalpractice doi:10.1038/ncpcardio1148 confirmed by the demonstration of the persistent ability to increase abnormally low heart rate in models of sinus node dysfunction by preserving the physiological response to autonomic modulation.

Another area that has produced promising results is 'molecular ablation', achieved by the exogenous administration of DNA to modify regional expression of ion channels to realize a specific functional objective. For example, augmenting the inward rectifier potassium current $\left(I_{\mathrm{K} 1}\right)$ by the overexpression of channel components could be used to create a local increase in refractoriness and prevent propagation of electrical impulses from arrhythmic foci. This approach could represent the next generation of ablation, in which 'DNA delivery' replaces 'tissue burning' to create an exit block.

The abbreviation of prolonged repolarizations has been attempted by several investigators. This therapeutic technique has provided encouraging results that could in future be applied to patients with long QT syndrome or those with heart failure in whom prolonged repolarization is arrhythmogenic. It should be kept in mind, however, that the transmural heterogeneity of ion channels in the heart poses a real challenge to transgenic delivery of 'electrical' proteins and that cardiac delivery of genes aimed at shortening action potential duration could turn into a proarrhythmic intervention.

Irrespective of the approach selected to modify the substrate of arrhythmias, it is clear that technical advances in vector technology and in their delivery still represent the major obstacle for development of cardiac gene therapy. When a nontoxic, nonimmunogenic, cardiac-specific transfer vector that allows long-term expression of the therapeutic sequences becomes available, and when effective delivery methods ensure that 'off target' treatment is avoided, gene therapy for arrhythmias will be ready for prime time. 